\section{Particle Physics in Budapest}

At the Conference on Particle Physics held at Budapest, July 4-9, an unusually large amount of new and exciting experimental results was presented.

Certainly an outstanding highlight was the discovery of a very heavy dimuon resonance with mass around $9.5 \mathrm{GeV}$ in the process $\mathrm{p}+$ nucleus $\rightarrow$ $\rightarrow \mu^{+} \mu^{-}+$anything. The experiment had been performed at the Fermi National Accelerator Laboratory with $400 \mathrm{GeV}$ protons by a ColumbiaFNAL-Stony Brook collaboration. This new state at $9.5 \mathrm{GeV}$ probably indicates the existence of at least a fifth quark (flavour), just as the previously discovered $\mathrm{J} / \Psi$ resonances announced the existence of the fourth quark (flavour): charm. Due to the limited resolution of the experiment, it is yet an open question whether this enhancement around $9.5 \mathrm{GeV}$ is a single (broad) resonance or - as predicted theoretically - consists of several narrow positronium-like bound states of the new quarks.

Far-reaching new results came from charged current deep inelastic $v$ and $\bar{v}$ interactions. These experiments have been performed at the new CERN-SPS accelerator and at FNAL. A previous experiment (Harvard-Penn-Wisconsin-FNAL collaboration) had claimed to see an anomalous change in certain $\bar{v}$ distributions for $\bar{v}$ energies beyond $30 \mathrm{GeV}$. This anomaly has a strong consequence : it had to be interpreted as a manifestation of right-handed currents coupling to new quarks beyond charm of mass around $5 \mathrm{GeV}$. In particular the new high statistics $v$ and $\bar{v}$ experiment from CERN (CERN-Heidelberg-Dortmund-Saclay collaboration) has clearly demonstrated now that such an $\bar{v}$ anomaly does not exist. Moreover all $v$ and $\bar{v}$ results are in nice agreement with the naïve spin $1 / 2$ quark-parton model (possibly adorned with some "asymptotic freedom" corrections). This CERN experiment and the other experiments performed at the big CERN bubble chamber $B E B C$ and at FNAL agree with each other.

Important progress came again from $\mathrm{e}^{+} \mathrm{e}^{-}$physics : first, a new $\Psi$ resonance with a mass of $3772 \mathrm{MeV}$ has been discovered at the SPEAR $\mathrm{e}^{+} \mathrm{e}^{-}$colliding beam machine. This state is a particularly interesting member of the $\mathrm{J} / \Psi$ family ("charmonium") since it is located just above the threshold for charmed D meson pair production and below the threshold for production of its excited state $D^{*}$. This peculiar kinematical configuration has made it possible to extract highly accurate and important information on the $D$ meson from the decay of this new $\Psi$ (3772) state.

Second, from the DORIS $\mathrm{e}^{+} \mathrm{e}^{-}$colliding beam machine came first evidence for the strange member of the charmed pseudoscalar meson family : the $\mathrm{F}$ meson. All these results round off beautifully our picture of the rôle of the fourth quark (flavour): charm.

A large amount of new (indirect) evidence for the heavy lepton $\tau$ has accumulated from the DORIS and SPEAR $\mathrm{e}^{+} \mathrm{e}^{-}$machines. The new results are in nice agreement with the theoretical expectations for such a heavy lepton.

A series of three fairly narrow peaks (at 1498, 1820 and $2130 \mathrm{MeV}$ ) has been reported from the $\mathrm{e}^{+} \mathrm{e}^{-}$colliding beam facility ADONE. The masses are consistent with expectations for higher spin one $\varnothing$ meson excitations. However, the reported widths seem somewhat too narrow, so perhaps there is interesting new physics about to emerge.

Even in the domain of "old" physics (i.e., neither involding charm nor quarks beyond charm) there was interesting and intriguing news: two groups working at the CERN Omega spectrometer have reported the discovery of new heavy baryon-antibaryon resonances at $2020 \mathrm{MeV}, 2200 \mathrm{MeV}$ and $2950 \mathrm{MeV}$ with surprisingly narrow widths. They are candidates for the predicted "baryonium" states. Theoretically one expects a somewhat longer lifetime for "baryonium" than for normal mesonic resonances due to the topological peculiarity of baryons as composites of three quarks. The puzzle with the new states is that their widths are considerably smaller than may be expected in such schemes.

In the domain of "theoretical theory" much effort was devoted to a better understanding of quark dynamics in general and the quark confinement problem in particular. Interesting new developments in the field of "supergravity" and in the investigation of non-perturbative solutions of classical and quantum field theories were reported.

A special highlight was certainly' the beautiful and inspiring lecture by Prof. Dirac celebrating the 50th anniversary of the Dirac equation at this Budapest Conference.

F. Schrempp

\section{THE UNCERTAINTY PRINCIPLE AND FOUNDATIONS OF QUANTUM \\ MECHANICS}

edited by W.C. Price and S.S. Chissick both of King's College, London.

This commemorates the formulation of quantum (or matrix) mechanics by Heisenberg, and collects topics into four main groups dealing respectively with Uncertainty Relations, their history and philosophical implications, particularly on causality,

Measurement Theory, Formal Quantum Theory and Applied Quantum Mechanics.

590 pages

0471994146

\section{VIBRATIONAL STATES}

by S. Califano, University of Florence.

Fulfils a need for a general theoretical introduction to vibrational spectroscopy at a graduate level, but includes topics of a more advanced character for the benefit of research workers.

348 pages

0471129968

April 1976

\section{OPTICS OF THIN FILMS}

by $\mathbf{Z}$. Knittl, Palacky University, Czechoslovakia.

Describes the basic theory of dielectric, metallic and inhomogeneous (dielectric) layers. The matrix treatment is the general method of approach, but other existing methods, including the graphical ones, are also followed. (Wiley Series in Pure and Applied Optics).

548 pages

$047149531 X$

INTRODUCTORY EIGENPHYSICS: An Approach to the Theory of Fields by C.A. Croxton, Cambridge.

286 pages

0471189294

286 pages

November 1974

0471189308 f8.65/\$17.30 (cloth)

November 1974 £4.95/\$9.90 (paper)

\section{INTRODUCTION TO LIQUID STATE PHYSICS}

by C.A. Croxton, Cambridge.

296 pages

0471189332

296 pages

0471189340

April 1975

£9.50/\$19.00

April 1975

£4.95/\$9.90

\section{NUCLEAR ELECTRONICS}

by P.W. Nicholson, University of London.

402 pages

0471636975

April 1974

£10.90/\$21.80 